\title{
Computing a Collision-Free Path using the monogenic scale space
}

Karl Holmquist, Deniz Senel and Michael Felsberg

The self-archived postprint version of this conference article is available at Linköping University Institutional Repository (DiVA):

http:/ / urn.kb.se/ resolve?urn=urn:nbn:se:liu:diva- 152713

N.B.: When citing this work, cite the original publication.

Holmquist, K., Senel, D., Felsberg, M., (2018), Computing a Collision-Free Path using the monogenic scale space, 2018 IEEE/ RSJ International Conference on Intelligent Robots and Systems (IROS), , 8097-8102. https:// doi.org/ 10.1109/ IROS.2018.8593583

Original publication available at:

https:/ / doi.org/ 10.1109/IROS.2018.8593583

Copyright: IEEE

http:// www.ieee.org/

(C) 2018 IEEE. Personal use of this material is permitted. However, permission to reprint/ republish this material for advertising or promotional purposes or for creating new collective works for resale or redistribution to servers or lists, or to reuse any copyrighted component of this work in other works must be obtained from the IEEE. 


\title{
Computing a Collision-Free Path using the monogenic scale space
}

\author{
Karl Holmquist ${ }^{1}$ and Deniz Şenel ${ }^{2}$ and Michael Felsberg ${ }^{1}$
}

\begin{abstract}
Mobile robots have been used for various purposes with different functionalities which require them to freely move in environments containing both static and dynamic obstacles to accomplish given tasks. One of the most relevant capabilities in terms of navigating a mobile robot in such an environment is to find a safe path to a goal position. This paper shows that there exists an accurate solution to the Laplace equation which allows finding a collision-free path and that it can be efficiently calculated for a rectangular bounded domain such as a map which is represented as an image. This is accomplished by the use of the monogenic scale space resulting in a vector field which describes the attracting and repelling forces from the obstacles and the goal. The method is shown to work in reasonably convex domains and by the use of tessellation of the environment map for non-convex environments.
\end{abstract}

\section{INTRODUCTION}

Many mobile robot applications require planning of a safe path that leads a robot to a goal position from a starting point. A common task of a mobile robot is to navigate through an indoor environment [1]. The task can be vacuum cleaning of an apartment, delivery of items in hospitals, schools, libraries, supermarkets, surveillance, etc. In all of these applications, the challenge is to design the algorithm that generates an obstacle-free path from a robot's start position to a goal position. Such planning includes intermediate steps such as building the map of the environment [2], finding an obstacle free path to the target position, and optionally optimization of the path.

A common analogy for using potentials-based path finding algorithms is that obstacles are acting repelling on the robot as parts with equal electrical charge, diffusing as per the heat equations [3] or as a ball rolling in the inside of a bowl. It can also be explained as the flow of water with obstacles modelled as rocks. Both of these analogies choose to model the environment as a nonconvex space since the obstacles are seen as outside of the work-space. As such they use boundary conditions such as the Neumann or Dirichlet conditions [4] to enforce a geodesic or similar distance measure that models a propagation inside of the non-convex space. This creates a problem which is generally hard to solve and requires approximate solutions.

\footnotetext{
${ }^{1}$ Karl Holmquist and Michael Felsberg are with the Department of Electrical Engineering, Computer Vision Laboratory, Linköping University, Sweden karl.holmquist@liu.se

${ }^{2}$ Deniz Şenel is a visiting researcher with the Department of Electrical Engineering, Computer Vision Laboratory, Linköping University, Sweden deniz.sevis@boun.edu.tr
}

By relaxing these kinds of boundary conditions to only be valid on the edge of the work-space, or rather the map itself, and see the obstacles as part of the work-space. We show that this relaxation allows for an efficient calculation of a path which is useful even in more complex environments. Fig. 1 shows a flow chart of the method, from input map to final path.

\section{A. Related Work}

There exist various methods in the literature for mobile robot path planning and each method has its own strengths and weaknesses. Potential field methods have been very popular and are widely used for path planning applications [3], [5], [6]. There are also sampling based methods that use a grid map of the environment [7], [8], [9]. Another popular technique is the combination of potential values and grid map of the environment as in [10], [11]. Our method does not generate a set of paths to follow in the map as in [10], instead it calculates the complete field for a region allowing fast calculation of a path from any position. This allows for applying a motion model or use of momentum to find a smooth trajectory for the robot to follow. An explicit creation of grids of the environment is not necessary since the method is valid for continuous maps as well. The implementation, however, assumes that the map has been sampled beforehand.

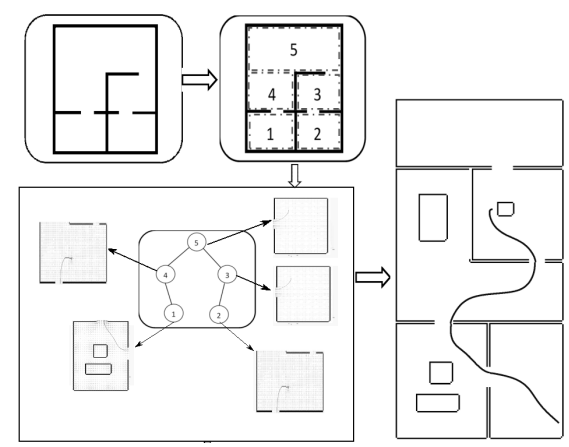

Fig. 1: Flow chart of the proposed algorithm.

Another approach which is commonly used in diverse areas is the $A^{*}$-algorithm [12]. This algorithm explores a state-space, by using a heuristic over which direction is the most beneficial to explore first, while trying to find the most cost-efficient path to the terminal state. However, the shortest path is often dangerous, since it will pass close to obstacles and requires consideration. A comparison between our proposed method and an $A^{*}$ implementation has been done and the effect of 
augmenting the heuristic of the $A^{*}$-algorithm with the potential field generated by using the monogenic scale space is also presented.

Meadow maps or navigation meshes [13] can at first glance appear similar to tessellating the environment into smaller convex regions. However, our aim is to create larger areas with obstacles and possibly varying cost functions inside of the same cell allowing for more flexibility in the actual shape and construction of the tessellated areas.

Minkowski sums [14], more commonly known as dilations, are used to create safety margins in path planning. This is a pre-processing step which requires a convolution over the edges of all obstacles. Our method does this step implicitly while also generating a vector field describing repulsive force from the obstacle.

The medial axis algorithm [15] generates a skeleton of the environments resulting in paths that pass at a far distance from most obstacles. This can be easily used to produce safe passages, but if the start and ending point is not part of the axis a separate step needs to be taken to reach the goal.

A family of more modern algorithms is the $R R T^{*}$ or rapidly-exploring random tree family [16]. The two-phase approach of $A^{*}-R R T^{*}$ [17] first performs a coarse graphsearch which is used to direct the $R R T^{*}$ search. Using this group of methods from the end configuration the tree can be reused for different starting locations, however, our proposed method can reuse parts of the calculated field even if both start and end configurations are changed.

\section{Mathematical Background}

Our method is based on the usage of a framework commonly used in computer vision: scale-space theory. Scale-space theory is used to represent a signal or image in multiple scales. In the case of images it gives an intuitive representation of how the image and its features appear at different scales by applying a so called kernel function to "smooth" the image.

We proposes to use the monogenic scale space, in turn based on the Poisson scale space, and therefore is a direct solution of the Laplace equation [18]:

$$
\Delta p(x, y, s)=(\nabla \cdot \nabla) p(x, y, s)=0 .
$$

The Laplace equation is commonly used in potential based path-planning [19]. The reason for its popularity is that the harmonic potentials that solves the equation, guarantees that no local minima will exist for a single point source. This property is called the min-max principle and guarantees that the maximum and minimum of the function is taken at the borders of the domain.

The Laplace equation can be solved on a convex domain (map) in an analytical way. The problem is however, hard to solve on a general non-convex domain which is often the case of ordinary environments, especially since that obstacles inside of a room can easily make the domain non-convex.
The Laplace equation has a number of fundamental solutions, The Poisson kernel being one. The Poisson kernel can be parameterized as in equation 2. This kernel is used in both the Poisson scale space as well as in the monogenic scale space and depends on two set of variables; $\mathbf{x}$, a n-dimensional vector containing the spatial coordinates. And $s$, the scale factor in the scale space. In most usages the number of dimensions will be 2 such that, $\mathbf{x}=(x, y)$.

$$
P_{s}(\mathbf{x})=\frac{s}{2 \pi\left(|\mathbf{x}|^{2}+s^{2}\right)^{3 / 2}}
$$

The monogenic scale space is a $(n+1)$-dimensional vector valued scale space while the Poisson scale space is a scalar scale space. The real part of the monogenic scale space, $p_{s}(\mathbf{x})$ and the imaginary vector part, $\mathbf{q}_{s}(\mathbf{x})$, creates the monogenic scale space as follows, $f_{M}(\mathbf{x}, s)=$ $\left(p_{s}, \mathbf{q}_{s}\right)(\mathbf{x})$.

While the real part, $p_{s}(\mathbf{x})$, is the same as in the Poisson scale space. The imaginary vector valued part is calculated using the conjugated Poisson kernel, $Q_{s}(\mathbf{x})$.

$$
Q_{s}(\mathbf{x})=\frac{\mathbf{x}}{2 \pi\left(|\mathbf{x}|^{2}+s^{2}\right)^{3 / 2}}
$$

The conjugated Poisson kernel is similar to the Poisson kernel but differs in the numerator. This difference arises from the two kernels being the partial derivatives with regards to $\mathbf{x}$ and $s$, of the same function. This gives the property that while $P_{s}$ describes the filtered image at the scale $s$. The conjugated kernel, $Q_{s}$, gives the image "flow" or the direction and the magnitude of the diffusion border.

The two parts, $p_{s}(\mathbf{x})$ and $\mathbf{q}_{s}(\mathbf{x})$, of the monogenic scale space at a certain scale, $s$, is found by the convolution between the image and the Poisson kernel $P_{S}(\mathbf{x})$ as well as its conjugated kernel $Q_{s}(\mathbf{x})$.

$$
\begin{aligned}
f_{M}(\mathbf{x}, s) & =\left(p_{s}, \mathbf{q}_{s}\right)(\mathbf{x}) \\
p_{s} & =\left(f * P_{s}\right)(\mathbf{x}) \\
\mathbf{q}_{s} & =\left(f * Q_{s}\right)(\mathbf{x})
\end{aligned}
$$

The solution using this formulation is an exact way to calculate the solution to the Laplace equation and the vector field related to this on an infinite $n$-D space. It can either be implemented by a convolution or, possibly more efficiently, be calculated in the Fourier domain [20].

On a bounded domain this will only give an approximate solution if implemented using the Fast Fourier transform (FFT) since it implies a periodic repetition of the signal where the right-side is placed next to the left and similarly for up/down. This can possibly result in a flow that exits the domain leading the robot to unknown areas.

By changing the bounding condition to the Neumann boundary condition (see eq. 7), the solution can be found exactly on a bounded rectangular 2-D domain with the use of the Discrete Cosine and Sine transforms. [20] 


$$
\left.\mathbf{n} \cdot \nabla p(\mathbf{x}, s)\right|_{x \in \partial \Omega}=0
$$

Where $\partial \Omega$ is the edge of the domain and $\mathbf{n}$ is the normal vector to the edge in the point $\mathbf{x}$.

Modeling the environment with a set of point sources and point sinks as done in our approach will result in that the Laplace equation cannot guarantee that no local minima is present. This is because the superposition of solutions to the Laplace equation is not necessary an exact solution for the equation itself [19].

We show that even though the solution is not without local minima, it can still be used in relatively complex environments. Using the tessellation of the environment can allow the usage in arbitrary environments.

\section{A. Physical interpretation}

Since the monogenic scale space is a harmonic function, we know that both the divergence and the curl of the field is zero. This is one of the fundamental properties of magnetic fields as well, giving rise to closed loops for all magnetic flow lines. One common model for calculating the magnetic field of a permanent magnet is called the Gilbert model. In this model the magnetic field is viewed as generated by a lot of magnetic "charges" which are smeared along the poles. By using this model we can therefore use the edges of the obstacles as our "sources" rather than the entire obstacles. This acts a form a regularization of the method since it will remove the effect from differing border thickness between walls and the border of the domain.

\section{Path Planning Method}

In this work, the goal is to find an obstacle-free path such that a mobile robot can travel from a starting position to a goal position in an indoor environment by simply following the vector field generated as part of the Monogenic scale space of the environment. The map is represented by an grey-scale image indicating the free-space and obstacles, such as walls. Since it is represented as an image it will not be a perfect representation of the real environment but rather a sampled version of it. The map may have been created earlier by a robot surveying the area or it might be based on an architectural floor map of the area. In this study, simulations are performed on an artificial map as well as one map from the video game Dragon Age Origins (DAO), from the MovingAI data set [21]. However, the proposed method can be adapted to a continuous real map of an environment. An example map of an indoor environment with boundary and walls represented using black solid lines are illustrated in Fig. 2a.

We will in this section describe our proposed method and the outline of it can be seen in algorithm 1 .

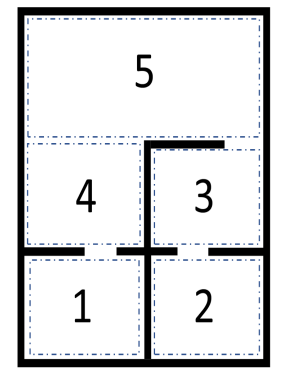

(a) An example map

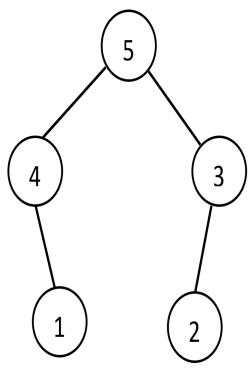

(b) Graph representation of the map
Fig. 2: Tessellation of an example map with maximal rectangles and its graph representation

\section{A. Graph Representation of the Environment}

The proposed method uses a hybrid maprepresentation, combining the use of a graph for describing how different sub-parts of the map is connected by doors, elevators and stairs. An example representation can be seen in Fig. 2. The graph used is an undirected graph with potentially multiple edges between the same nodes and cycles. Allowing for a simple representation of indoor environments. Each node is represented by a sub-map containing the part of the map it covers. In the case of non-axis aligned walls or curved walls, it can also contain surrounding space since the sub-maps are allowed to overlap. The edges also holds information regarding where the opening between the areas are and can be chosen to represent the whole passage between areas or a smaller part of for example a door-way to further direct the robot toward a certain part of it.

\section{B. Tessellation of the Environment}

The tessellation of the map is responsible for creating the graph as well as separate the map into sub-regions. We assume that such a tessellation already exists which in many cases is true when talking about architectural floor-maps. In the experiments a manual tessellation of the environment has been done by finding a bounding box which contains most of the room, except when the form of the room itself is highly non-convex as the Lshaped room in Fig. 3 .

\section{Finding and Calculating the Path}

Finding the global path is done in steps. First, a nodeto-node path is found to limit the necessary potential fields that needs to be calculated. Secondly, a local path for each node is calculated, connecting the entrance or start to the exit or goal position. Finally, the local paths are merged into a single global path connecting start and goal.

1) Finding a Path in the Graph: Calculating a path through a simple graph can be done in a variety of ways. Since the number of nodes of the graph generally is low while the cost of passing through a specific room 
is unknown before calculating the local path, any graph search method that can handle cycles in an undirected graph is sufficient.

2) Calculating the Path Inside Each Node: The calculation of the local path is the main point of our approach, using the monogenic scale space to find a continuous path through the local environment.

The general steps in the algorithm can be seen in algorithm 1. Calculating two distinct monogenic scale spaces, one for the obstacles at scale $s_{o}$ and another only containing the goal or the exit of the area at scale $s_{g}$, allows for a linear combination of these to assure that the robot does not pass through obstacles. This approach also allows for reusing the same monogenic scale space generated from the obstacle if the same area is revisited with a different goal.

In order to combine these two scale space signals, $\left(p_{o}, \mathbf{q}_{o}\right)$ and $\left(p_{g}, \mathbf{q}_{g}\right)$, for obstacles and the goal respectively. We want the importance of the obstacles to be the mayor influence close to obstacles and otherwise a combination of the two. This is accomplished by calculating a weight mask for every pixel, see algorithm 2

Using the vector field $\mathbf{q}$, the path is calculated by stepwise calculate the local flow at the current position and taking a step in that direction. The resulting path is that is commonly referred to as a streamline.

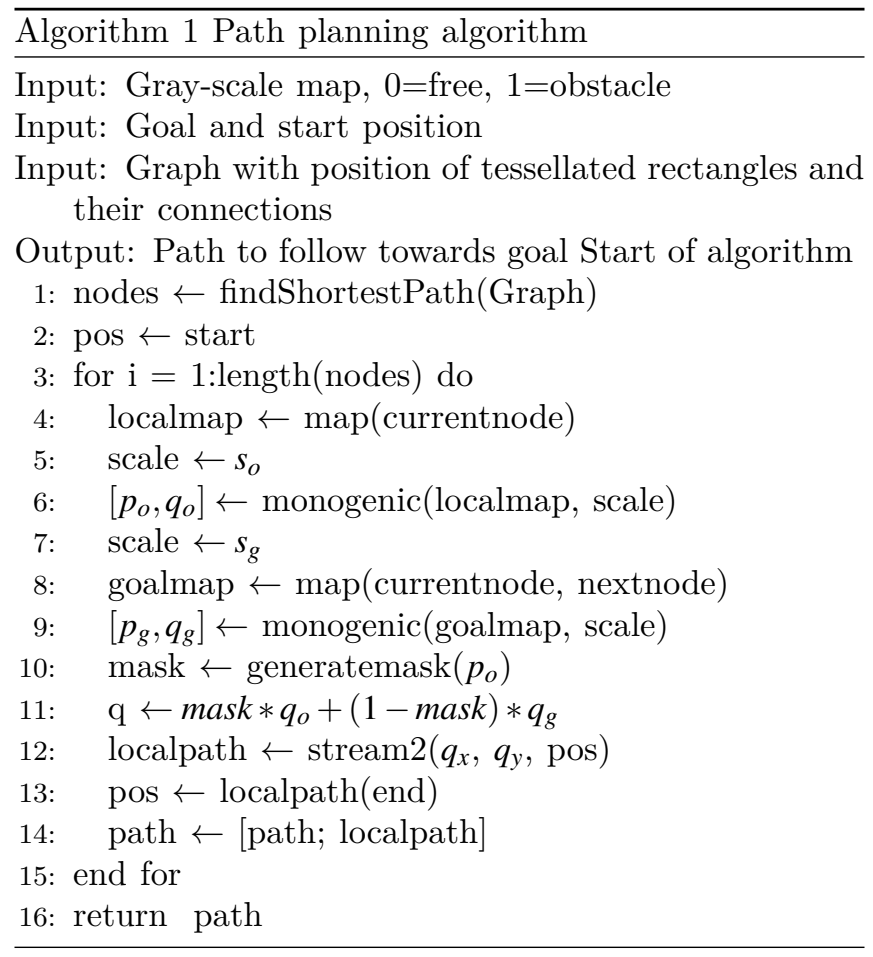

Fig. 3: First scenario: Rooms with a single obstacle, original map in background

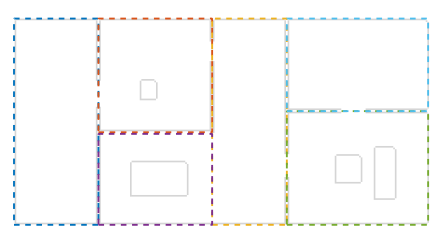

Fig. 4: Map showing the tessellated environment.

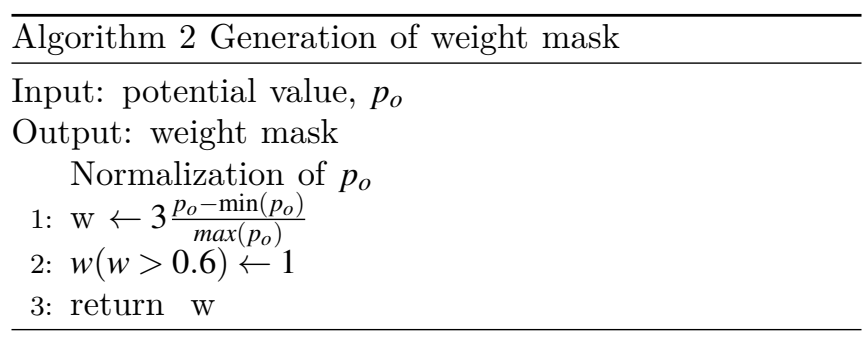

\section{Simulation Results}

In this section, different scenarios are used to demonstrate the performance of the proposed algorithm. For this purpose, first we apply the proposed method to a scenario where the mobile robot navigates through rooms to reach its goal position using an artificially created map. Two different scenarios are considered: rooms with few or many obstacles. Secondly, an example map from the MovingAI [21] dataset is used to show how the proposed method performs in a more complex environment. Finally, an obstacle-free map of the same environment is used to compare a direct approach of finding a path using an uninformed $A^{*}$-algorithm or which equals the Dijkstra algorithm as well as an informed $A^{*}$-algorithm [12] using the real part of the Monogenic signal as an exploration cost.

\section{A. Artificial map with differing degree of obstacles}

In the first example given in Fig. 3, the starting position of the robot is in the left room while the terminal position is in the middle of the lower middle room on the map. The calculated path using the proposed algorithm can be seen in Fig. 3, indicated by a blue line.

In the second example, there are more obstaclesplaced in some rooms whose contours can be seen in Fig. 5. This time, the starting position is in the upper middle room and goal is the corner of the top right room in Fig. 5 . As can be seen, the robot can reach the goal position without colliding with walls or any of the obstacles as indicated by the blue path in Fig. 5. The tessellation of the original map before creating the potential field can be seen in Fig. 4. Here gray lines and rectangles are walls and obstacles, and the coloured dashed lines marks the different tessellated areas. 
Fig. 5: Second scenario: rooms with multiple obstacles inside, detected edges of the original map in background

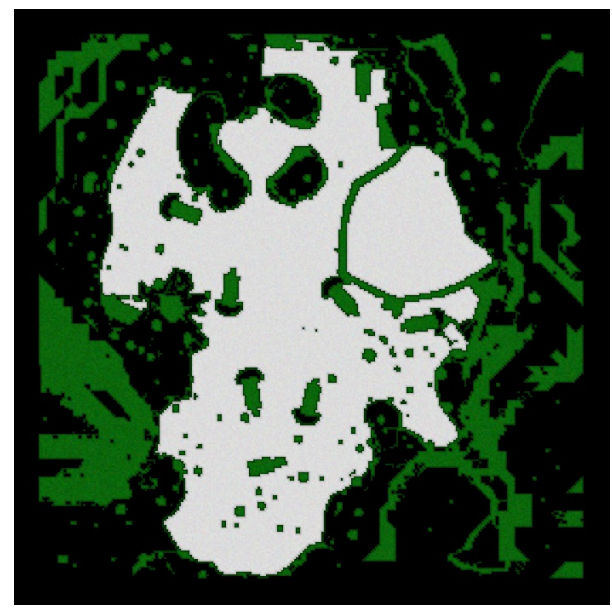

Fig. 6: Example scenario of a more complex environment from the Movingai dataset [21]

B. Evaluation without tessellation in a complex environment

In the interest of seeing the robustness of the algorithm in more complex environments we used one of the maps (named brc504d.map) from the computer game Dragon Age Origins. But instead of using the relatively low resolution map $(256 \times 256)$ with grid-based notation of terrain types as tree, passable and impassable. We use the image in Fig. 6 of the environment and thresholds it to only keep passable and impassable, e.g. setting all trees as impassable. This results in a $700 \times 700$ image instead (the image used is the thumbnail available at the homepage of the dataset).

From this image we randomize a set of trajectories which are only limited by requiring start and end positions to be in passable terrain. From these example trajectories we chooses two which we deem to be informative showing a bit of what kind of situations can be handled without tessellating the map.

A path from the top to the bottom is shown in Fig. 6 which also shows how it manages to pass through a

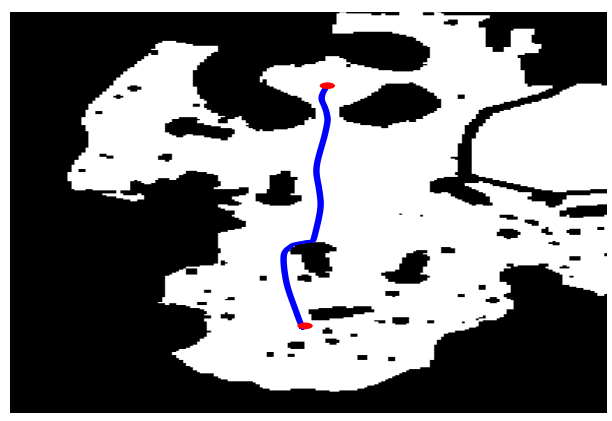

Fig. 7: Example of successfull path

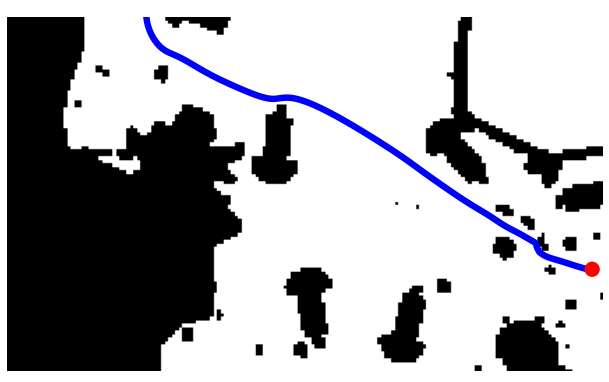

Fig. 8: Example of successfull path

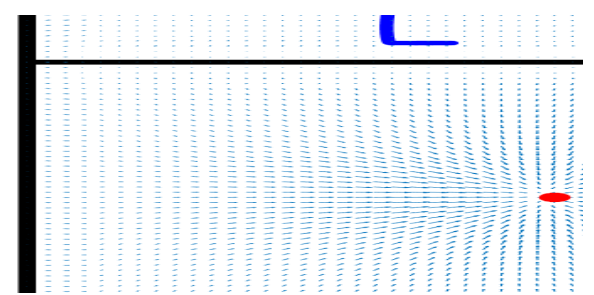

Fig. 9: Example of when the algorithm fail because of highly non-convex environment between start and goal position, blue arrows illustrate the flow lines.

relatively small passage-way at the top.

Fig. 8 shows a path traversing the map from left to right. It shows how the found path curves around the obstacles, both larger and smaller. In both figures the path is passing apparently close to some of the obstacles, this is easily configurable by changing the scale, $s_{o}$, used in the algorithm.

The example in Fig. 9 shows a situation which the direct approach is insufficient because of the non-convexity of the local area. In this kind of situations, there is necessary using a tessellation of the map as we have proposed or some kind of way-points separating the path into parts which can be found.

For completeness, the following parameters was used in the DAO examples: the scale of the monogenic scale space of the obstacle map, $s_{o}=1$ and for the goal map, $s_{g}=60$. The map size was as mentioned earlier $700 \times 700$.

\section{Comparative study}

We also performed a comparative study using one of the most famous methods for path planning in discretized spaces, the $A^{*}$-algorithm. The $A^{*}$-algorithm was limited to search in a the 8-neighbourhood. Two different heuristics and costs were used for the $A^{*}$ algorithm, the first was completely uninformed resulting in the shortest possible path or Dijkstra's algorithm. The second used the potential field, $p$, generated by the help of the monogenic scale space as proposed in out method.

These two variants of the $A^{*}$ was compared to the usage of streamlines based on the vector field generated in our method. The different paths found in an example map is shown in Fig. 10. And the relative runtimes of the methods and the length of the paths are shown in table If. 


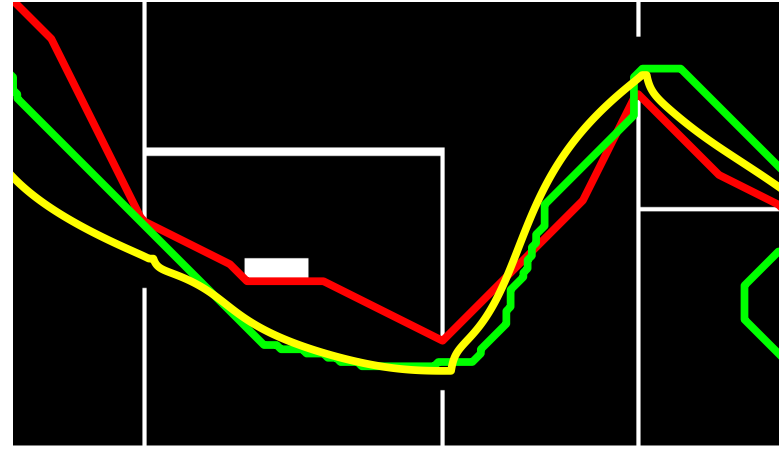

Fig. 10: Comparison of path using Dijkstra (red), $A^{*}$ (green) and our method (yellow)

TABLE I: Comparison in run-time and length of found path.

\begin{tabular}{|l|ll|}
\hline \multicolumn{2}{|l|}{ length (pix) } & Time (ms) \\
\hline Dijkstra & 323.8 & 295 \\
Monogenic method & 375.0 & 42 \\
- Preprocessing of map & - & 5 \\
Monogenic method $+A^{*}$ & 393.6 & 592 \\
$-A^{*}$ & - & 550 \\
\hline
\end{tabular}

As can be seen in the figure and from the distance measure, the Dijkstra algorithm results in the shortest path but it also passes directly next to many of the obstacles and walls, resulting in what should be seen as a dangerous path.

On the otherhand, both the informed $A^{*}$-algorithm and the proposed method are avoiding the obstacles with a margin. This results in longer paths but allows for imprecision in the control and positioning of the robot.

Comparing the times should be done with care since neither approach has been optimized and are run in Matlab on a Laptop. However, we can see the tendency that even with the extra preprocessing steps of the map necessary for our method, the time is significantly lower than the Dijkstra algorithm. Even taking into considerations that Matlab is well optimized for matrix operations which is most of the parts of our method, $42 \mathrm{~ms}$ in processing time is small since the potential map can be reused during multiple visits as well as shared between multiple agents.

The monogenic scale space approach gives also the added benefit of an actual vector field which can be used for finding a continuous paths. Using the $A^{*}$-algorithm is generally limited to discrete positions.

\section{Conclusion}

In this paper we propose a potential based hybrid path-planning approach, using the structure of the environment to separate it into simpler regions. This tessellation allows using an efficient solution for the Laplace equation as well as avoiding some of the risks with potential field methods, such as local minima. As the simulation results reveal, it is possible to find a safe path even in the most crowded parts of an environment and it is possible to adjust how close the path should be to the obstacles by just changing the scale used in the algorithm. As the comparative study suggests, the proposed method finds a safer path since the path found using it is not as close to the obstacles as the path found using Dijkstra's algorithm.

\section{Acknowledgements}

This work was founded by the European Union's Horizon 2020 Programme under grant agreement 644839 (CENTAURO).

\section{References}

[1] S. Thrun, "Learning metric-topological maps for indoor mobile robot navigation," Artificial Intelligence, pp. 21-71, 1999.

[2] J. J. Leonard and H. F. Durrant-Whyte, "Simultaneous map building and localization for an autonomous mobile robot," in Proc. IEEE IROS '91.'International Workshop on Intelligence for Mechanical Systems, Osaka, Japan, Nov. 1991, pp. 14421447.

[3] Y. Wang and G. S. Chirikjian, "A new potential field method for robot path planning," in Proceedings 2000 ICRA. Millennium Conference. IEEE International Conference on Robotics and Automation. Symposia Proceedings (Cat. No.00CH37065), vol. 2, 2000, pp. 977-982 vol.2.

[4] R. Chen, C. Gotsman, and K. Hormann, "Path planning with divergence-based distance functions," arXiv preprint arXiv:1708.02845, 2017.

[5] M. G. Park and M. C. Lee, "Artificial potential field based path planning for mobile robots using a virtual obstacle concept," in Proc. IEEE ASME International Conference on Advanced Intelligent Mechatronics, 2003, pp. 735-740.

[6] Y. K. Hwang and N. Ahuja, "A potential field approach to path planning," IEEE Trans. Robot. Automat., vol. 8, pp. 23-32, feb. 1992.

[7] D. Ferguson and A. Stentz, "Field d*: An interpolation-based path planner and replanner," Robotics Research, pp. 239-253, 2007.

[8] S. Thrun and A. Bücken, "Integrating grid-based and topological maps for mobile robot navigation," in Proc. National Conference on Artificial Intelligence, Oregon, Aug. 1996.

[9] F. Duchoň, A. Babinec, M. Kajan, P. Beňo, M. Florek, T. Fico, and L. Jurišica, "Path planning with modified a star algorithm for a mobile robot," Procedia Engineering, vol. 96, pp. 59-69, 2014.

[10] S. Garrido, L. Moreno, D. Blanco, and P. Jurewicz, "Path planning for mobile robot navigation using voronoi diagram and fast marching," International Journal of Robotics and Automation, vol. 2, pp. 42-64, 2011.

[11] M. Soulignac, "Feasible and optimal path planning in strong current fields," IEEE Trans. Robot., vol. 27, pp. 89-98, feb. 2011.

[12] R. Dechter and J. Pearl, "Generalized best-first search strategies and the optimality of a*," J. ACM, vol 32, no. 3, pp. 505-536, July 1985, [Online]. Available: http: //doi.acm.org/10.1145/3828.3830

[13] R. C. Arkin, "Path planning for a vision-based autonomous robot," in Mobile Robots I, vol. 727. International Society for Optics and Photonics, 1987, pp. 240-251.

[14] E. Oks and M. Sharir, "Minkowski sums of monotone and general simple polygons," Discrete \& Computational Geometry, vol. 35, no. 2, pn. 223-240, Feb 2006. [Online]. Available: https://doi.org/10.1007/s00454-005-1206-y

[15] O. Aichholzer, W. Aigner, F. Aurenhammer, T. Hackl, B. Jüttler, and M. Rabl, "Medial axis computation for planar free-form shapes," Computer-Aided Design, vol. 41, no. 5, pp. 339 - 349, 2009, voronoi Diagrams and their Applications. [Online]. Available: htt.t://www.sciencedirect. com/science/article/pii/S0010448508001681 
[16] S. Karaman and E. Frazzoli, "Sampling-based algorithms for optimal motion planning," The international journal of robotics research, vol. 30, no. 7, pp. 846-894, 2011.

[17] M. Brunner, B. Brüggemann, and D. Schulz, "Hierarchical rough terrain motion planning using an optimal samplingbased method," 2013 IEEE International Conference on Robotics and Automation, pp. 5539-5544, 2013.

[18] M. Felsberg and G. Sommer, "The monogenic signal," IEEE Trans. Signal Processing, vol. 49, pp. 3136-3144, Dec. 2001.

19] C. I. Connolly, J. B. Burns, and R. Weiss, "Path planning using laplace's equation," in Robotics and Automation, 1990. Proceedings., 1990 IEEE International Conference on. IEEE, 1990, pp. 2102-2106.

[20] M. Felsberg, R. Duits, and L. Florack, "The monogenic scale space on a bounded domain and its applications," in International Conference on Scale-Space Theories in Computer Vision. Springer, 2003, pp. 209-224.

[21] N. Sturtevant, "Benchmarks for grid-based pathfinding," Transactions on Computational Intelligence and AI in Games, vol. 4, no. 2. pn. $144-148$, 2012. [Online]. Available: http://web.cs.du.edu/ sturtevant/papers/benchmarks.pdf 